\title{
Balkanologie
}

Revue d'études pluridisciplinaires

Vol. $16 n^{\circ} 1 \mid 2021$

Au-delà de la « route des Balkans »: mondes sociaux des circulations

\section{Les expériences d'une ville industrielle française vécues par les ouvriers yougoslaves dans les années 1960-2000 à Sochaux-Montbéliard}

The Experiences of a French Industrial City by Yugoslav Workers in Sochaux-

Montbéliard in the Years 1960-2000

Juliette Ronsin

\section{(2) OpenEdition}

Journals

Édition électronique

URL : https://journals.openedition.org/balkanologie/3023

DOI : 10.4000/balkanologie.3023

ISSN : 1965-0582

Éditeur

Association française d'études sur les Balkans (Afebalk)

Référence électronique

Juliette Ronsin, «Les expériences d'une ville industrielle française vécues par les ouvriers yougoslaves dans les années 1960-2000 à Sochaux-Montbéliard», Balkanologie [En ligne], Vol. 16 n 1 | 2021, mis en ligne le 01 juin 2021, consulté le 03 octobre 2021. URL : http://journals.openedition.org/ balkanologie/3023 ; DOI : https://doi.org/10.4000/balkanologie.3023

Ce document a été généré automatiquement le 3 octobre 2021.

(c) Tous droits réservés 


\section{Les expériences d'une ville industrielle française vécues par les ouvriers yougoslaves dans les années 1960-2000 à Sochaux- Montbéliard}

The Experiences of a French Industrial City by Yugoslav Workers in SochauxMontbéliard in the Years 1960-2000

Juliette Ronsin

1 La ville de Montbéliard, en Franche-Comté, et son agglomération, le Pays de Montbéliard, ont parfois été qualifiées de "nébuleuse ${ }^{1}$ ", au sein de laquelle se trouve l'ancien village de Sochaux. Le Pays de Montbéliard est marqué par la forte emprise des dynasties industrielles Peugeot et Japy sur le contexte local. Dans les années 1960, Peugeot a été confronté à un manque d'effectif et a recruté davantage d'ouvriers immigrés, en passant par l'intermédiaire de l'office national d'immigration (ONI). Les profils des ouvriers ainsi embauchés étaient spécifiques : il s'agissait d'hommes jeunes, souvent célibataires et sortant du service militaire. Ces recrutements de l'ONI concernaient des Portugais, des Marocains, des Yougoslaves ou des Turcs. Après la signature d'un accord bilatéral entre la France et la Yougoslavie le 25 janvier 1965, de nombreux Yougoslaves ont été embauchés par Peugeot à Sochaux. Ils furent jusqu'à 4880 dans le Pays de Montbéliard en 1973 et constituaient alors la nationalité la plus représentée parmi les ouvriers étrangers dans les années $1970^{2}$. Le nombre très important de Yougoslaves dans le Pays de Montbéliard est une particularité par rapport à d'autres régions françaises et à d'autres bassins d'emploi ${ }^{3}$ et justifie que l'on s'y intéresse davantage.

2 Alors que l'immigration yougoslave en France a fait l'objet de synthèses globales à l'échelle nationale ${ }^{4}$, je propose d'analyser le cas des immigrés et immigrées yougoslaves à une échelle plus locale et m'intéresserai à leurs expériences d'une ville industrielle en 
France, Sochaux-Montbéliard, où les Yougoslaves étaient à la fois immigrés et, en grande majorité, ouvriers. Étant donnée cette double appartenance, il nous semble intéressant d'analyser leur appropriation de la ville et de l'espace en s'inspirant des travaux portant sur les rapports entre les ouvriers et la ville, en particulier ceux d'Olivier Schwartz sur des ouvriers d'une ancienne région minière du Nord de la France dans les années $1980^{5}$. Ce travail sera aussi réalisé à la lumière des recherches portant sur l'exclusion des ouvriers de l'espace urbain, telle qu'abordée par des philosophes comme Henri Lefebvre dans son ouvrage éponyme sur le "droit à la ville ${ }^{6}$ ", ou encore Michel Verret qui analyse la culture ouvrière et questionne le « droit à l'espace ${ }^{7}$ ». Dans les deux cas, l'emprise sur l'espace de la ville s'apparente à un droit, à un bien commun.

3 Ces travaux résonnent d'autant plus dans le contexte particulier qui nous intéresse : à Sochaux-Montbéliard, le développement de l'agglomération autour de l'ancien village de Sochaux est directement lié aux activités de l'entreprise Peugeot, mais aussi aux aménagements de ce qui était alors le District urbain du pays de Montbéliard (DUPM) ${ }^{8}$. Le DUPM a en effet également contribué à aménager les communes de l'agglomération et a joué un rôle complémentaire à celui de l'entreprise Peugeot. À Sochaux, la fonction productive et industrielle de la ville se trouve alors surreprésentée, aux dépends d'autres types d'activités. Dans cet espace urbain fortement marqué par l'industrie et organisé autour de ces unités de production, comment les immigrés yougoslaves, ouvriers, ont-ils développé leurs pratiques de la ville?

4 À partir d'extraits d'entretiens avec d'anciens ouvriers et ouvrières originaires d'exYougoslavie arrivés en France dans les années 1960-1970, de documents d'archive concernant les logements des immigrés ainsi que de dossiers d'étrangers provenant des Archives départementales du Territoire de Belfort, cet article aborde les trajectoires d'ouvriers ex-yougoslaves des années 1960 à nos jours dans leur lien avec l'espace de Sochaux-Montbéliard. Les existences des ouvriers yougoslaves étant souvent connectées à la Yougoslavie, grâce aux appels téléphoniques ou aux vacances passées l'été dans la ville ou le village familial, cet article se penche sur l'expérience de l'espace de Sochaux-Montbéliard en tenant compte de la dimension transnationale des trajectoires migratoires. 
Fig. 1. Le District urbain du pays de Montbéliard (DUPM), ancêtre du Pays de Montbéliard Agglomération (PMA)



\section{De la Yougoslavie à la France : la ville et la découverte du Pays de Montbéliard}

5 S'il s'avère difficile de distinguer les motivations économiques et politiques présidant au départ de Yougoslavie, on peut affirmer que le peu de perspectives d'avenir ressenties par la jeunesse dans un pays en proie à un chômage très fort ${ }^{9}$ était l'une des raisons principales de partir vers un autre pays dans les années 1960-1970. Le rêve d'un mode de vie à l'occidentale, dans la "société de consommation", l'idée d'offrir de meilleures conditions de vie matérielle à sa famille et la volonté de partir loin pouvaient également contribuer à motiver ces choix.

6 L'espace de la ville apparaissait comme le plus à même de répondre à ces attentes : avant même de quitter la Yougoslavie, les futurs ouvriers s'étaient déjà déplacés vers des grandes villes yougoslaves, où s'effectuait le recrutement par les entreprises, françaises en particulier, et les visites médicales, souvent encadrées par l'ONI, en fonction des besoins de main-d'œuvre des entreprises. La ville était ainsi associée au développement, à l'emploi et à la modernité, tandis que, par opposition, les espaces ruraux du sud de la Serbie - dont étaient originaires de nombreux Yougoslaves embauchés par Peugeot - pouvaient être associés à des représentations négatives. Des interprètes et professeurs venus de Belgrade ou du nord de la Serbie ont souvent calqué une vision binaire d'un Nord plus cultivé et d'un Sud pauvre et moins dynamique ${ }^{10}$. Pour reprendre la terminologie de l'historienne Maria Todorova, ces stéréotypes peuvent être qualifiés de «balkanismes ${ }^{11}$ » et renvoient au passé ottoman du sud de la 
Serbie, davantage associé à des représentations de moindre développement économique. Pour ces interprètes et professeurs immigrés, il s'agit alors de déplacer l'altérité vers d'autres Yougoslaves, tandis que l'ensemble de la Yougoslavie faisait l'objet de ces clichés en France. Cette volonté de se distinguer s'explique aussi par un différentiel social, dépassant l'idée d'homogénéité sociale des Yougoslaves dans le Pays de Montbéliard appartenant alors surtout à la classe ouvrière.

Une fois arrivés en France, les conditions de logement des ouvriers immigrés étaient, dans un premier temps, plutôt improvisées ${ }^{12}$, avant la construction de logements plus imposants. Devant l'omniprésence du béton, certains Yougoslaves étaient nostalgiques de leur ancien village. C'est le cas de la fille d'un immigré de Voïvodine, arrivée en France alors qu'elle était enfant :

Donc je suis arrivée dans le Pays de Montbéliard, à Étupes, dans des immeubles à

douze étages, on était au sixième et ça c'était mon premier choc ${ }^{13}$.

Željko, fils d'ouvrier yougoslave - devenu lui-même ouvrier par la suite -, arrivé en France quand il était enfant, s'exprime en ces termes :

Et puis là quand je suis arrivé en étant gamin, je me rappelle très bien, quand je regardais comme ça les bâtiments, j'avais la tête qui tournait, tellement que c'était haut, tellement que c'était grand ${ }^{14}$.

9 Face à la déception, l'espoir d'un retour prochain en Yougoslavie permettait de tenir, tout comme on supportait les conditions de travail, mieux rémunéré mais particulièrement difficile, dans les fonderies, comme en témoigne un ancien ouvrier de Peugeot ${ }^{15}$ :

La plupart, enfin une bonne partie des gens qui sont venus là-bas [dans les usines de Peugeot], ils sont venus plus c'était payé [pour une paye élevée]. La paye était plus importante en fonderie, peinture. [...] Ils se battaient pour travailler là-bas, gagner plus l'argent et envisageaient comme tous de partir. C'était pas le cas, on est tous restés.

10 L'espace de la ville, dans le Pays de Montbéliard, était alors envisagé comme provisoire car il restait toujours la possibilité de repartir après avoir économisé. Par la suite, cet espace a pu progressivement apparaitre comme un espace d'enfermement, si les choses ne se passaient pas comme prévu.

\section{L'usine, au cœur des spatialités ouvrières}

11 Les années 1960-1980 correspondent à la période la plus dynamique à SochauxMontbéliard. Jusqu'à la fin des années 1970, les usines Peugeot tournaient à plein. Pour les ouvriers yougoslaves, le quotidien était marqué par le travail et quelques échappatoires, comme les moments de loisir ou les vacances en Yougoslavie l'été.

Pour convaincre les futurs embauchés, l'entreprise Peugeot vantait les mérites et le dynamisme de la région, à seulement quelques kilomètres de la Suisse, de l'Allemagne et de Paris. Or, le terme d'«enclave» revient souvent pour désigner le pays de Montbéliard ${ }^{16}$. Une enclave naturelle tout d'abord, d'où il est difficile de sortir, avec les limites que constituent les massifs du Jura et des Vosges. L'enclave est aussi celle d'une ville marquée par les activités d'une entreprise paternaliste, avec notamment l'ancien village de Sochaux qui s'est développé autour des activités de Peugeot. L'espace a été aménagé par l'entreprise, jusqu'aux supermarchés et aux compagnies de bus, d'où une sensation de surveillance permanente pour les ouvriers. D'après les données des 
registres d'inscription du personnel, on a pu calculer que les ouvriers yougoslaves embauchés en 1972 étaient logés dans les lieux suivants : l'hôtel Peugeot de Grand Charmont (37\%), l'hôtel Peugeot d'Audincourt (35\%), l'hôtel Peugeot de Sochaux $(20 \%)$ et enfin le foyer AMAT d'Audincourt $(6 \%)^{17}$. Ces logements, gérés par l'entreprise Peugeot ou des associations, comme l'Association montbéliardaise d'aide aux travailleurs (AMAT), étaient donc situés dans des villes proches de l'usine. À Sochaux, l'hôtel Peugeot où se trouvaient les Yougoslaves était principalement l'hôtel $\mathrm{n}^{\circ} 1$, dont les bâtiments furent construits à partir de 1928.

Fig. 2. À Sochaux : à gauche les immeubles de dortoirs et à droite l'hôtel Peugeot $n^{\circ} 1$

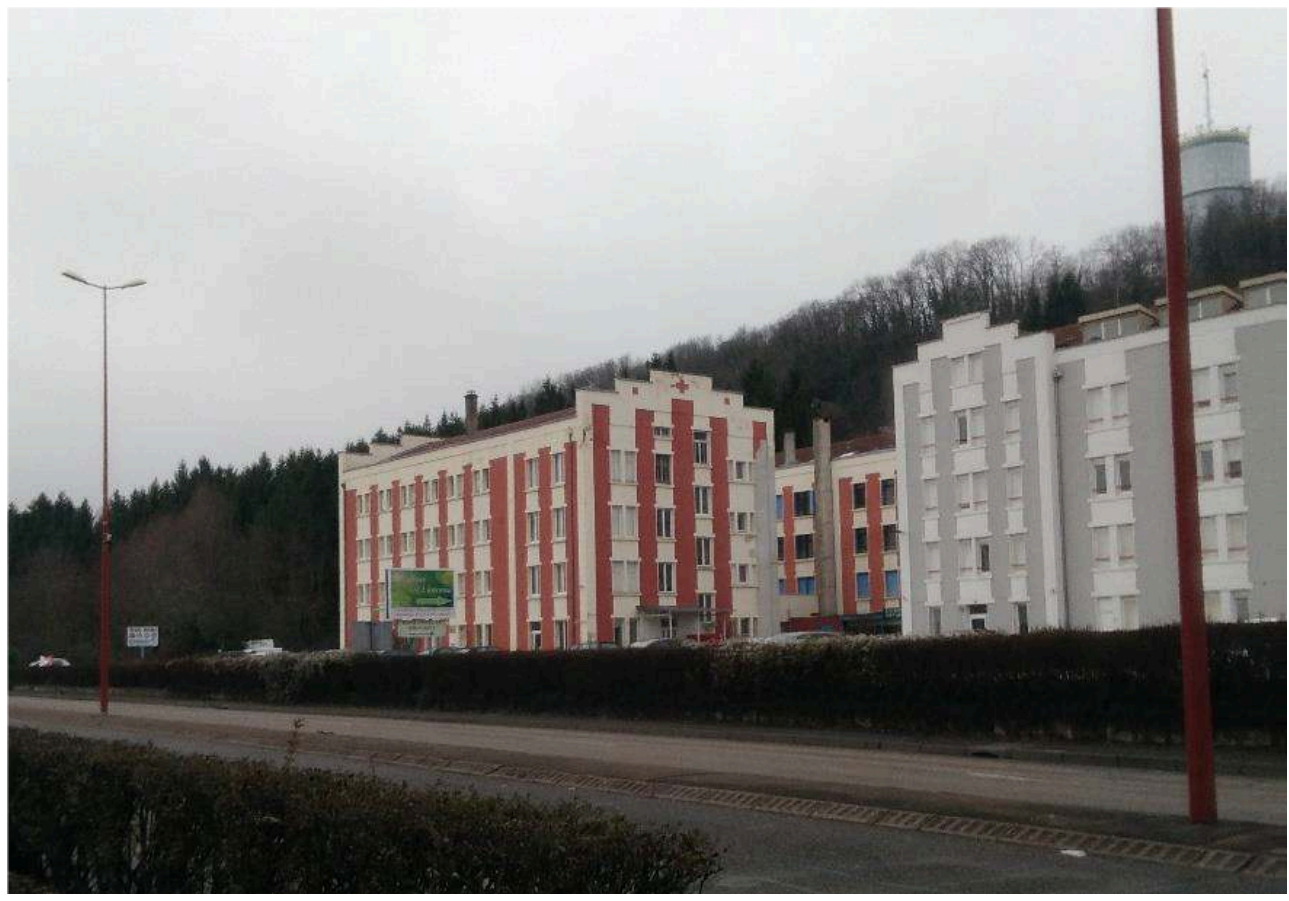

Photographie de Juliette Ronsin, 2018

L'éloignement de ces bâtiments instaurait une coupure avec la ville et avec les personnes d'autres nationalités, logées dans d'autres foyers ou hôtels Peugeot. On en trouve notamment la trace dans des notes produites par la sous-préfecture de Montbéliard en 1969, évoquant "l'inconvénient d'une forte concentration ethnique dans les Hôtels Peugeot ${ }^{18}$ ».

Des frontières invisibles de classes se sont ainsi renforcées dans l'organisation de la ville, avec des logements pour travailleurs isolés des quartiers de villas destinées aux cadres de l'entreprise. L'espace séparant le logement du lieu de travail était essentiellement traversé pour les allers et venues à l'usine en autocars, au sein desquels la répartition se faisait également par nationalités et par foyers d'habitation ${ }^{19}$. Sochaux était alors une ville dynamique, avec des bruits incessants de voitures, de motocyclettes et d'usines. On retrouve cette atmosphère étourdissante dans les films de la série Le lion, sa cage et ses ailes, tournés à Sochaux à la fin des années 1970 par Armand Gatti et des ouvriers de différentes nationalités, dans le but de combattre les préjugés racistes ${ }^{20}$. Le temps de la ville était rythmé par le travail à l'usine, qui s'effectuait à toute heure de la journée et de la nuit, selon les horaires de roulement, et certains lits d'hôtel 
pouvaient être partagés successivement par différents ouvriers en fonction de leurs horaires.

\section{Tentatives d'émancipation et contrôle}

15 Dans cette vie rythmée par le travail à l'usine, certains espaces de la ville faisaient l'objet de tentatives d'appropriations ouvrières. Les ouvriers étaient alors regroupés entre hommes ${ }^{21}$ dans les usines ou les foyers d'habitation et cela se poursuivait lors des sorties hors de l'usine, dans les bars en particulier. Comme on le voit dans le film documentaire « Sochaux : 9000 célibataires » réalisé en 1971 pour l'ORTF, il était alors nécessaire de "descendre en ville " pour trouver des activités de loisir ou faire «tous les bistrots ", selon les mots d'un jeune ouvrier interrogé. La voix-off du film poursuit de cette manière : « Il y a chez ces jeunes gens une espèce de mélancolie, de vague à l'âme, d'ennui, tout proche du vide intérieur dont ils ne sont pas responsables. C'est le drame des heures creuses que les animateurs des foyers de jeunes travailleurs connaissent bien. Les cités où se trouvent ces foyers ont grandi trop vite : on n'y trouve ni cinéma, ni piscine, ni club, ni stade. Il en résulte que les jeunes de ces foyers vivent dans un monde $\operatorname{clos}^{22}$ ». À ce cloisonnement spatial s'ajoutait un fort risque d'enfermement social. Dans les démarches quotidiennes avec la Sécurité sociale, les difficultés à s'exprimer en français pouvaient conduire à l'isolement et la frustration. De plus, la menace d'un retour en Yougoslavie en cas de mauvaise santé planait en permanence.

marge de manœuvre était réduite pour les travailleurs immigrés, certain ouvriers yougoslaves tentèrent de quitter les foyers pour des logements individuels dans les environs, dans le Territoire de Belfort par exemple. Ces mobilités résidentielles étaient parfois très importantes. Ainsi, en un an, entre le 3 janvier 1973 et le 19 janvier 1974, un ancien ouvrier de Peugeot a vécu dans cinq villes différentes, en passant par Grand-Charmont (Doubs), Grandvillars (Territoire de Belfort), Paris, Besançon (Doubs), Delle (Territoire de Belfort) et Danjoutin (Territoire de Belfort) ${ }^{23}$. La possession du permis de conduire et d'un véhicule s'avérait alors indispensable pour se déplacer vers d'autres villes industrielles proches de taille moyenne, comme Belfort ou Mulhouse, et pour trouver un travail ${ }^{24}$. Cela constituait encore un obstacle à la mobilité de ces ouvriers venus de Yougoslavie. D'après des informations trouvées dans une lettre d'un commissaire de police envoyée au préfet de la région de Franche-Comté, préfet du Doubs, nous apprenons que l'« échange systématique et sans contrôle d'un permis de conduire yougoslave contre le brevet équivalent français" a été suspendu le 12 décembre 1970. De plus, des formulaires vierges étaient alors en vente libre en Yougoslavie et c'était «au candidat à l'examen de se procurer en librairie-papeterie le brevet qu'il fera remplir et viser en cas de réussite ${ }^{25}$ ». À partir de 1970, le permis de conduire yougoslave n'étant plus systématiquement échangé contre un permis français, la possession d'un permis de conduire français constituait une difficulté administrative supplémentaire pour les ouvriers yougoslaves ${ }^{26}$.

17 Au-delà des moyens d'accès à la mobilité, tous les déplacements d'étrangers, même entre villes proches, étaient contrôlés par les services administratifs et de police, donnant lieu à la constitution de dossiers individuels d'étrangers. En effet, au moment des demandes de renouvellement de visas dans de nouvelles villes d'habitation, des enquêtes de moralité et de police étaient réalisées et le renouvellement du visa était 
conditionné par l'exercice d'un emploi en France, après les circulaires MarcellinFontanet ${ }^{27}$. Ces déplacements résidentiels, dont la recherche d'emploi était souvent la cause, s'accompagnaient aussi de changements professionnels. Après avoir travaillé chez Peugeot, l'ouvrier ayant déménagé cinq fois en un an a ainsi, dans un premier temps, exercé l'emploi de manutentionnaire à Besançon. Ces nouveaux emplois étaient souvent trouvés dans des usines de métallurgie, produisant parfois des pièces pour Peugeot, ou dans des entreprises de maçonnerie. Cette mobilité professionnelle ouvrière était d'ailleurs redoutée par l'entreprise Peugeot, qui craignait que les ouvriers de l'entreprise ne partent travailler de manière informelle dans des entreprises concurrentes pour l'embauche de la main-d'œuvre. Ainsi, dans des notes dactylographiées datées de 1969, produites par la sous-préfecture de Montbéliard et ayant pour titre «Problèmes posés par l'emploi de la main-d'œuvre yougoslave ", il est indiqué que «la délivrance du permis de séjour de six mois correspondant à la durée des contrats de travail permet d'éviter que les travailleurs yougoslaves arrivés en fin de contrat aux Automobiles PEUGEOT se répandent dans la Région et soient une proie facile pour certains employeurs tout prêts à les embaucher à du travail "noir"28 ".

\section{Installation et appropriation de l'espace}

Les trajectoires résidentielles des Yougoslaves ont souvent évolué de l'hôtel pour travailleurs célibataires aux logements HLM et aux pavillons, en raison notamment de la dimension familiale de ces parcours. Le regroupement familial fait l'objet d'une visibilité nouvelle et est autorisé par le décret du 29 avril 1976. Il correspond cependant à une réalité ancienne. De nombreuses femmes avaient ainsi rejoint leur époux à Sochaux-Montbéliard, parfois avec des enfants, six mois ou un an après l'arrivée de ceux-ci en France. Pour les familles de Yougoslaves du Pays de Montbéliard, les lieux d'habitation succédant aux logements provisoires étaient souvent d'imposantes barres de HLM, comme on l'observe dans le «Film yougoslave» d'Armand Gatti avec l'évocation d'un immeuble qualifié de "mur des Yougoslaves ${ }^{29}$ ». Sur ces images, tournées depuis la fenêtre de l'un de ces bâtiments, les vis-à-vis sont importants et la vue sur d'autres bâtiments imposants et gris est le seul horizon. Plusieurs familles vivaient ainsi dans le quartier des Buis, à Valentigney, où existait alors une certaine convivialité, comme en témoigne un extrait d'entretien d'Ana, arrivée en France quand elle était enfant ${ }^{30}$ :

Je devais avoir 8 ans quand on est arrivés vivre aux Buis. Et puis après oui ça, à 10 ans je commençais à être intégrée, à ce que ça aille mieux à l'école, avoir des copains des copines. Mais c'est-à-dire que pendant deux ans c'était un petit peu chaotique. Et ensuite, bah aux Buis il y avait de tout : des Espagnols, des Portugais, des Marocains, où c'était la super entente, je me suis super... où je trouvais ça génial. Mes parents étaient bien aussi parce qu'ils vivaient entre eux. Alors pour eux, ils avaient plus l'impression... ils étaient moins perdus.

Après avoir vécu dans des lieux où il n'y avait aucun immigré et où la xénophobie était perceptible, ceux qui arrivaient dans ce nouveau quartier avaient accès à des sociabilités nouvelles, permettant de se comprendre, de partager des expériences communes, prolongées lors d'excursions dans la campagne des alentours, tandis que les cours de serbo-croate ${ }^{31}$ étaient par exemple l'occasion pour les enfants et les parents de se retrouver. 

Valentigney, se sont distingués des autres habitants en achetant un pavillon. Or, le
choix de rester dans le même quartier peut être interprété comme un attachement fort
à ce lieu, mais aussi comme une volonté d'y montrer leur réussite sociale.

Fig. 3. Le « mur des Yougoslave»

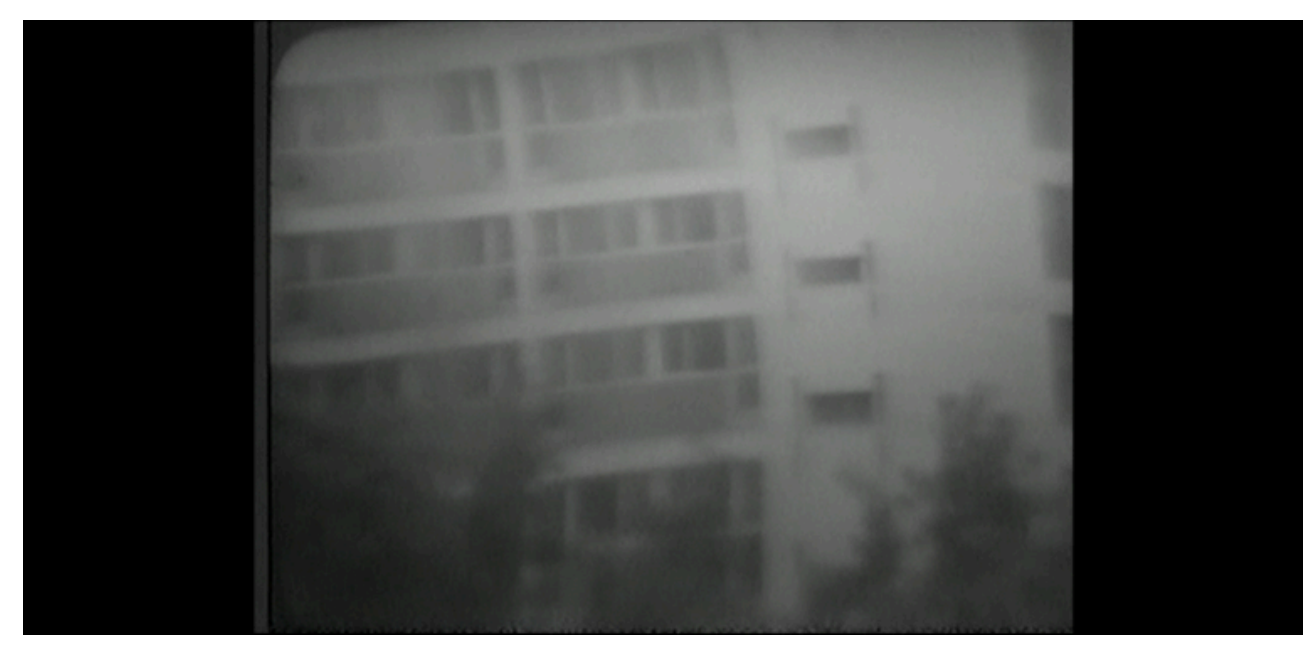

Extrait du film d'Armand Gatti, Le lion, sa cage et ses ailes, 1976.

\section{La recherche d'échappatoires}

Par contraste avec la "ville-usine ${ }^{34}$ », la campagne environnante apparaissait comme un espace de fuite recherché. Le week-end était un moment propice aux excursions en voiture. La proximité de Montbéliard avec les frontières suisse et allemande donnait l'opportunité de se rendre aisément dans ces pays. La voiture permettait alors de conquérir des espaces de liberté, tandis qu'elle était associée à une certaine souffrance lorsqu'elle était construite à l'usine. Avec la possibilité de posséder une voiture Peugeot grâce aux réductions de prix proposées par l'entreprise à ses employés, ces représentations s'inversaient. Néanmoins, du fait d'excès de vitesse, les cas d'accidents de voiture ont laissé des traces dans les archives. En consultant les dossiers d'étrangers, on trouve ainsi le cas d'un ouvrier de Peugeot, résidant dans un hôtel Peugeot, décédé à 22 ans dans une voiture Peugeot ${ }^{35}$.

Dans les entretiens avec des retraités qui étaient ouvriers dans les années 1960-1970, on note une mobilité liée aux loisirs à cette époque. Certains disent : " pendant ces années, on a bien profité ", en évoquant les balades et les excursions, même si ce constat est peut-être embelli eu égard à la situation actuelle. Avec l'arrivée des familles dans le Pays de Montbéliard, des pique-niques entre amis étaient organisés dans la nature. La 
« nature » était alors perçue comme un espace moins transformé, sur lequel on pouvait enfin avoir une emprise et où le rapport au temps se relâchait. Les espaces verts même en ville - devenaient attrayants, notamment grâce aux activités de jardinage qui permettent de transformer l'espace à sa guise et d'exprimer sa créativitée ${ }^{36}$. Il s'agissait alors surtout de parcelles de jardins ouvriers louées, où s'opérait un retour aux activités agricoles exercées durant la jeunesse en Yougoslavie. Un ancien ouvrier, originaire d'une région rurale en Serbie, s'exprime en ces termes :

Ah mais nous on fait bio, on fait pas n'importe quoi. [...] Moi je pratique la chirurgie,

je suis chirurgien. Chirurgien botaniste ! ${ }^{37}$

24 Avec le jardinage, il est possible de valoriser un savoir-faire peu reconnu, du moins à l'usine ${ }^{38}$, et acquis avant l'arrivée en France. Cette activité est également empreinte d'une certaine nostalgie. Ces savoir-faire donnent aussi lieu à un sentiment de supériorité et à un constat : les « jeunes » ne savent plus bêcher la terre.

Le « retour à la terre » évoqué à propos des jardins ouvriers pouvait aussi se manifester lors des retours au village d'origine. La possession d'une voiture permettait de rentrer en Yougoslavie durant les congés. Or, dès leur retour en Yougoslavie, les personnes venues de France se faisaient remarquer. En effet, les expériences de la ville et des biens de consommation vécues en France étaient alors montrées de manière ostentatoire, avec l'apport de divers biens, comme de l'argent mais aussi des meubles, des sanitaires neufs ou des sculptures pour jardin. De la même manière, dans son étude sur les émigrés albanais originaires de la République de Macédoine dans les années 2000, l'historien Robert Pichler décrit la volonté de ces émigrés de montrer leur réussite économique en public, lors des retours dans les villages d'origine. Ces voyages sont aussi l'occasion de manifester une appartenance constante à la communauté villageoise ${ }^{39}$, en participant aux travaux des champs. Lors de ces séjours en Yougoslavie, le contraste perçu entre les villages familiaux et Sochaux est grand, surtout pour les enfants et petits-enfants d'ouvriers nés en France. Željko évoque ainsi les vacances en ex-Yougoslavie :

Même quand on part en vacances on voit la différence, c'est pas le même style de vie. Ici on a notre train-train. Par exemple, le matin ici pour le petit-déj', on prend du lait, des céréales, des gâteaux. Là-bas c'est comme en Angleterre, c'est des œufs, du lard. Et puis eux [les enfants] ils comprenaient pas ${ }^{40}$.

L'expérience de la ruralité en ex-Yougoslavie, dans des villages plutôt isolés où un certain mode de vie paysan est parfois toujours d'actualité, diffère alors fortement de celle de Sochaux, ville industrielle pourtant proche des espaces ruraux.

Par ailleurs, à Sochaux-Montbéliard, des lieux fixes où l'on peut se retrouver ont également émergé. Dans le Pays de Montbéliard, il n'existe pas un quartier ou une rue qui serait spécifiquement habitée ou fréquentée par les Yougoslaves, mais plutôt des îlots - tels certains cafés ou une épicerie de "produits balkaniques", connus par le bouche-à-oreille. Ces espaces peuvent aussi être mobiles, comme les salles louées pour certains bals, conduisant à des déplacements vers Belfort ${ }^{41}$ et vers l'Alsace, autour de Mulhouse $^{42}$, ou même en Suisse où des fêtes ont souvent lieu. Les cours de serbe pour les enfants sont d'ailleurs dispensés un jour dans la semaine par la même enseignante, à Mulhouse, Belfort et Montbéliard ${ }^{43}$. Un réseau s'est ainsi constitué progressivement depuis les années 1970 avec les villes de Belfort et Mulhouse où vivent également de nombreux ex-Yougoslaves ${ }^{44}$, et ce, malgré le ralentissement des activités de l'association franco-yougoslave, désormais franco-serbe ${ }^{45}$ dans le Pays de Montbéliard. Après le conflit yougoslave des années 1990, les lieux de sociabilité, comme les 
associations, se sont souvent scindés par nationalités. Les événements festifs, tout comme les cours de langue ${ }^{46}$, regroupent désormais des personnes originaires des mêmes États issus de l'éclatement de la Yougoslavie, alors que les différences entre les ressortissants des républiques yougoslaves étaient moins marquées auparavant.

\section{Vivre dans une région « en crise »}

28 À partir des années 1980, après le dynamisme des années 1960-1970, les activités industrielles du Pays de Montbéliard et de l'entreprise Peugeot connurent un ralentissement, qui s'accompagna d'un changement progressif du mode de production, caractérisé globalement par la transition du fordisme vers le toyotisme ${ }^{47}$. Comme le rappellent Stéphane Beaud et Michel Pialoux, après une période de forte baisse des effectifs des usines Peugeot, un regain d'activité a néanmoins été notable entre les années 1998 et $2001^{48}$, qui fut de courte durée. La restructuration des activités, avec un recours croissant à la sous-traitance, accompagnée de la fermeture de sites, caractérise davantage les années 2000-2010. Le travail sous forme de missions d'intérim et la soustraitance conduisent à des mobilités encore plus fortes et à un moindre ancrage dans l'espace de Sochaux. Dès la fin des années 1970, les contrats de travail des travailleurs immigrés n'étaient souvent pas renouvelés. D'une ville marquée par les allers et venues en mobylette des ouvriers à l'usine, on passa à une ville " en crise ", dans laquelle les activités économiques et de loisir se firent plus rares, comme l'évoque Ana :

Avant je me rappelle, quand on était au Buis, quand c'était l'heure où les bus de chez Peugeot arrivaient, on avait l'impression que c'était toute une ville qui sortait de ces bus. Y en avait quatre-cinq d'affilée, c'était rempli quoi. Et là je sais même pas si ça existe encore, parce que je sais pas s'ils le remplissent. Comme il y a plus que 1500 habitants aux Buis, alors que à l'époque, je sais même pas, on devait être 10000 facile. 1500 sur 10000 , vous vous imaginez comme la région ici elle a régressé. Donc je ne sais pas, chez Citroën, Renault, ça doit être pareil hein. Ça doit être la même chose ${ }^{49}$.

Le centre urbain de Montbéliard est désormais souvent délaissé au profit de villes aux alentours et de la Suisse, où les activités de loisir sont plus nombreuses, comme le mentionnent une ancienne ouvrière ex-yougoslave, Zorana, et sa voisine :

Zorana : Oh bah surtout ici, ici le coin là, c'est trop froid. Pas froid, attention, pas froid le froid, c'est les gens. [...] Et puis moi je comprends, maintenant les gens ils ont de plus en plus mal. Donc...

La voisine : Oui, puis surtout dépressifs.

Zorana : Voilà, oui.

La voisine : Ils ont plus de sous, tout le monde déprime. Moi, j'ai vu des copines là : « ah je prends du Lexomil, je prends... »... Oh j'ai dit, purée c'est pas après... Je lui ai dit «faut boire un coup! ». Là, j'en ai une, elle vit que par ses cachets. Je lui ai dit «bois un coup puis ça ira mieux $»^{50}$ !

\section{Transformations des activités et du paysage urbains dans les années 2000-2010}

Actuellement, si beaucoup d'ouvriers yougoslaves sont retournés en Yougoslavie ou profitent de la retraite pour partager leur vie entre la France et l'ex-Yougoslavie, ceux restés sur place vivent dans des résidences de type HLM, ou des pavillons. Ces lieux d'habitation se situent toujours autour et à proximité de Sochaux, ou dans des villes 
plus éloignées pour le pavillonnaire : dans les extrémités du pays de Montbéliard, voire en dehors du Doubs comme dans le Territoire de Belfort. Sur les images aériennes du Pays de Montbéliard, le site principal des usines de PSA est facilement identifiable à ses bâtiments industriels imposants, de forme géométrique. La ville-centre de Montbéliard se situe à l'ouest de Sochaux, tandis que le reste des habitations visibles est situé dans les autres villes de l'agglomération.

Fig. 4. Photographie aérienne d'une partie du Pays de Montbéliard

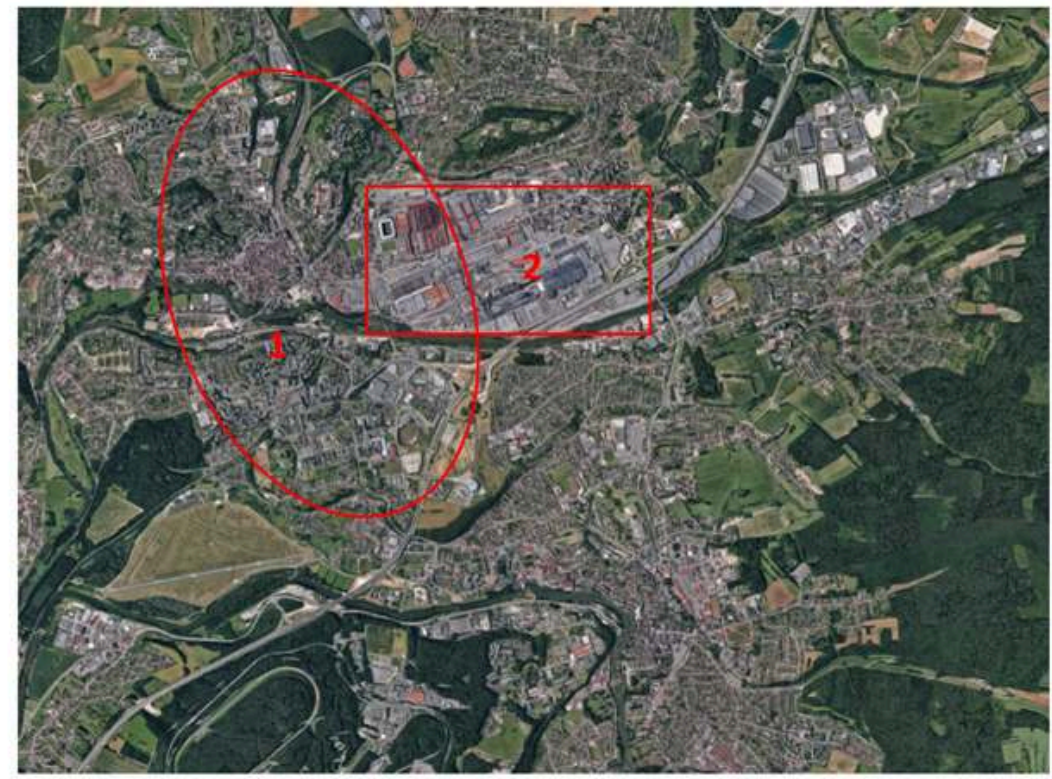

1 : ville de Montbéliard

2 : site principal des usines de PSA

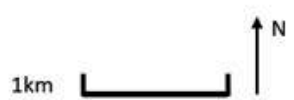

D’après Google, Image 2021.

31 De nombreux passages des entretiens réalisés ont trait aux représentations de l'espace dans le Pays de Montbéliard et à leur évolution sur une durée de près de cinquante ans, depuis l'arrivée en France. Ces repères spatiaux ont changé au fils du temps, suite aux évolutions urbaines.

Alors qu'un ancien ouvrier yougoslave, Dragan, évoque avec son voisin le lieu dans lequel il résidait quand il était ouvrier et célibataire, l'emplacement des foyers de résidence n'était pas exactement connu de son voisin présent lors de l'entretien, comme en témoigne cet extrait ${ }^{51}$ :

Dragan : Nan, c'était pas le foyer AMAT aux Forges [foyer pour travailleurs géré par l'Association montbéliardaise d'aide aux travailleurs], c'était le foyer... C'étaient les hôtels de Peugeot.

Le voisin: Ah oui. C'étaient, c'étaient les blocs bleus là, la SMCI [la SMCI est une société de promoteurs immobiliers originaire de Besançon].

Dragan : Oh là-bas, y avait plein de blocs. Vers la rivière là-bas.

Le voisin: Vers la rivière. Je vois. Enfin bon, Juliette elle peut pas voir, mais bon c'était, c'était... y avait des gens qui étaient logés dans des baraquements, dans des baraquements préfabriqués, hein, et puis y avait des gens qui étaient logés dans des foyers... 
Tandis que le voisin, un ancien ouvrier d'origine française également de chez Peugeot, ne situait pas l'emplacement de l'hôtel Peugeot d'Audincourt, l'évocation de la couleur des bâtiments permet de les identifier, dans l'uniformité où ils forment des blocs. Il est intéressant d'observer que l'ouvrier d'origine française ne connaissait pas précisément les foyers d'immigrés construits à la hâte dans les années 1960, difficilement différentiables et séparés spatialement dans l'espace urbain. Ses explications soulignent le fait que si l'on ne vient pas de la région, on ne peut pas " voir ", visualiser des repères spatiaux acquis localement. Entre les deux voisins, l'évocation d'éléments «naturels » sert de référent commun pour localiser immédiatement un lieu : la rivière (le Doubs ou un affluent ici) est largement connue et sert de point de repère. Sa simple mention permet alors de se rappeler des souvenirs anciens, comme ceux de terrains de football ou de bâtiments pour les travailleurs immigrés qui ont été détruits depuis :

Dragan : Bon quand on vient le matin, on vient du travail, on mange et puis certains qui se mettent au lit alors pour une heure ou deux. Et à 4 heures, comme ça, 5 heures et demie, 4 heures alors voilà, on sort, les équipes se forment. Et puis allez hop, jouer le football. Mais comme c'était près de la rivière alors des fois le ballon s'en va dans l'eau. (rire)

La mention de cette rivière contraste avec le côté très artificiel, vertical et imposant, des bâtiments, des blocs. Les bâtiments ont connu différents propriétaires mais, ce qui semble compter davantage, ce sont leur monumentalité et les souvenirs qui leur sont attachés.

Fig. 5. Wagons porte-automobiles transportant des voitures Peugeot neuves à la gare du centreville de Montbéliard

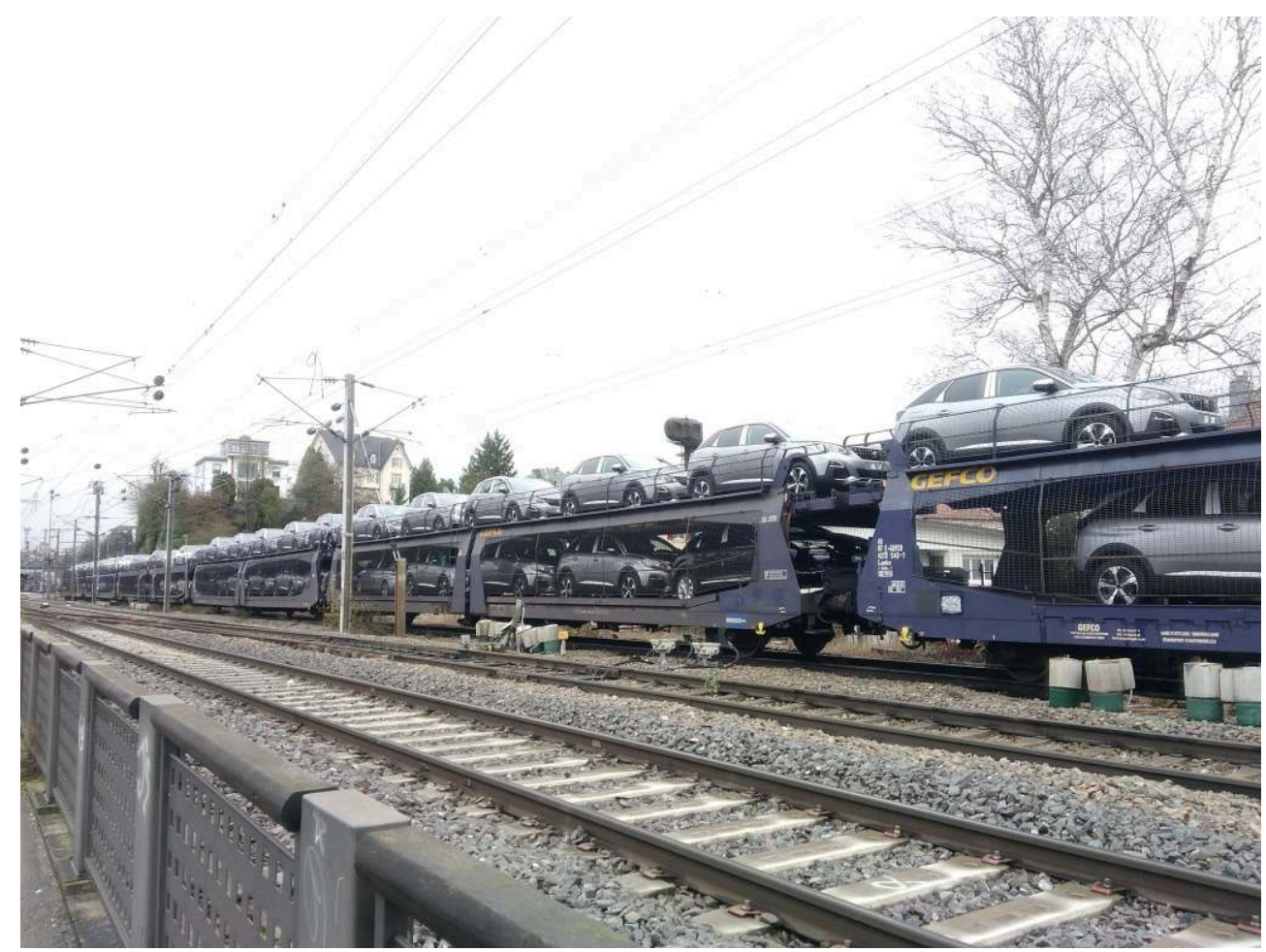

Photographie de Juliette Ronsin, 2018.

En arrivant à la gare du centre-ville de Montbéliard, la vision à perte de vue de wagons porte-automobiles Gefco, ancienne filiale de PSA, chargés de voitures Peugeot neuves 
destinées à être vendues dans toute la France rappelle l'activité industrielle toujours intense de Peugeot dans le Pays de Montbéliard. Néanmoins, le site même des usines de Peugeot à Sochaux a vu sa taille diminuer avec la destruction de plusieurs bâtiments. Le site de PSA connaît, ces dernières années, des phases de restructuration, auxquelles s'ajoute le projet "Sochaux 2022»: un bâtiment qui abritait l'atelier DS5 ${ }^{52}$ a par exemple été détruit et $62000 \mathrm{~m}^{2}$ de bâtiments vont encore être démolis, dont l'emblématique Cercle Hôtel. Des lieux comme la brasserie Peugeot, où a travaillé une famille retrouvée dans plusieurs sources d'archives, a par exemple fermé en 1979, et le bâtiment est désormais en ruine, devant le musée Peugeot flambant neuf.

L'affaiblissement de la condition ouvrière s'accompagne de la disparition de lieux de travail ; seuls les souvenirs - aussi imparfaits soient-ils - permettent de rappeler ces lieux, dont il ne reste plus de preuve visible dans les paysages, ou parfois des bâtiments abandonnés. Les quartiers industriels et populaires sont marqués par leur caractère éphémère, par opposition aux quartiers bourgeois davantage patrimonialisés. L'appropriation de l'espace public semble alors menacée sur le long terme du fait de la destruction d'espaces liés à des souvenirs marquants, le « droit à la ville » faisant l'objet de renégociations permanentes afin de trouver une place au sein de l'espace urbain.

\section{Conclusion}

Pouvoir se déplacer dans et en dehors de la ville constitue une forme de ressource. Même si, dans le Pays de Montbéliard, une grande partie de l'espace urbain a été aménagé et organisé par Peugeot, on peut observer plusieurs tentatives de négocier des espaces de liberté et de fuite : la fréquentation de bals, la recherche d'espaces naturels ou les excursions dans les régions environnantes. Pour reprendre à Michel Verret la notion de "droit à l'espace ", tout espace approprié par les ouvriers yougoslaves, audelà des hôtels Peugeot, apparaît bien ici comme « un espace pris sur l'oppression, donc un desserrement, si petit soit-il, de celle-ci ${ }^{53}$ ». À cela s'ajoutait, pour les Yougoslaves, la mobilité, et en particulier les voyages au pays qui permettaient de contrer l'ennui en constituant des coupures dans le temps, offrant un rythme qui n'est plus celui de l'usine. Cette analyse, partant de l'agglomération de Sochaux-Montbéliard, permet de souligner la non-linéarité des trajectoires avec des allers et retours fréquents entre Yougoslavie et France et des déplacements nombreux vers d'autres villes françaises. L'agglomération de Sochaux-Montbéliard constitue néanmoins un territoire associé à des événements marquants, le travail à l'usine et les souvenirs en France qui se sont constitués autour de cet espace. La "ville usine» est devenue aussi, dans certains quartiers, un espace de convivialité et de partage d'expériences communes, même si son existence et son devenir sont toujours soumis aux politiques de l'entreprise PSA et aux décisions des aménageurs de l'agglomération du Pays de Montbéliard.

Enfin, depuis les années 1990, de nouvelles personnes originaires d'ex-Yougoslavie sont également arrivées dans le Pays de Montbéliard : durant les conflits des années 1990, ce sont des réfugiées et réfugiés puis, depuis les années 2000, des personnes souvent jeunes à la recherche d'un emploi en Europe occidentale. Si ces mobilités n'ont pas nécessairement de lien avec celles des années 1970 - Peugeot n'est désormais plus une entreprise qui recrute massivement-, les nouveaux immigrés ex-yougoslaves se rendent souvent dans des villes où, comme à Sochaux, vivent des membres de leur famille ou des connaissances. 


\section{NOTES}

1. Le terme de «nébuleuse » est employé par Nicolas Hatzfeld et par Armand Gatti pour évoquer le Pays de Montbéliard. HATzFeld Nicolas, Les gens d'usine : 50 ans d'histoire à Peugeot-Sochaux, Paris, Éditions de l'Atelier, 2002, p. 7 ; GATTI Armand, « Film yougoslave : la bataille des trois P. (43') », Le lion, sa cage et ses ailes, France, série de 8 documentaires de 12 à 60 min, 1976, Paris, Montparnasse, cop. 2011.

2. En 1976, par exemple, les Yougoslaves représentaient $28,2 \%$ de la main-d'œuvre étrangère de Peugeot, suivis des Turcs (19,6\%) et des Algériens (18,3\%). Archives départementales du Doubs, 1569W308 : Logement des immigrés.

3. Dans l'entreprise Michelin, à Clermont-Ferrand, un autre bassin d'emploi où la présence des Yougoslaves était importante, les Yougoslaves ne constituaient que 11,7\% de l'effectif total en 1970. GUESLIN André (dir.), Les hommes du pneu : les ouvriers Michelin à Clermont-Ferrand de 1940 à 1980, Paris, Éditions de l'Atelier, 1999, p. 263.

4. Voir воsкоvic Sanja, «L'émigration yougoslave à Paris : entre intégration et exclusions ", Les Cahiers du MIMMOC, janvier 2006, en ligne: http://journals.openedition.org/mimmoc/167 (consulté en mai 2021) ; CACIC-KUMPES Jadranka, KUMPES Josip, «Approche socio-historique des migrations yougoslaves en France», Revue européenne des migrations internationales, vol. 6, $\mathrm{n}^{\circ} 2$, 1990, p. 65-84 ; MOROKVASIC Mirjana, « Des migrants “temporaires" : les Yougoslaves », Sociologie du travail, $\mathrm{n}^{\circ} 14-3,1972$, p. 260-277; MOROKVASIC Mirjana, Iz Jugoslavije na rad u Francusku [Les travailleurs yougoslaves en France], Zagreb, Centre de recherches sur les migrations, 1972; MOROKVASIC Mirjana, "Il était une fois une communauté des Yougoslaves en France", dans Laurent Gervereau, Pierre Milza, Emile Temime (dir.), Histoire de l'immigration en France au $X X^{e}$ siècle, Paris, Bibliothèque de documentation internationale contemporaine, Musée d'histoire contemporaine, Somogy, Éditions d'Art, 1998, p. 154 ; MOROKVASIC Mirjana, «Les Yougoslaves en France ", dans Laurent Gervereau, Yves Tomic, De l'unification à l'éclatement. L'espace yougoslave, un siècle d'histoire, Nanterre, Musée d'histoire contemporaine - BDIC, 1998, p. 160-165.

5. SCHWARTZ Olivier, Le monde privé des ouvriers: hommes et femmes du Nord, Paris, Presses universitaires de France, 1990.

6. LEFEBVRE Henri, Le droit à la ville, Paris, Éditions Anthropos, 1968.

7. VERRET Michel, L'espace ouvrier. L'ouvrier français, Paris, Armand Colin, 1979.

8. Créé en 1959, le DUPM est devenu en 1999 le Pays de Montbéliard Agglomération (PMA).

9. GOKALP Catherine, « L'émigration yougoslave », Population, nº 1, 1974. p. 25-60 (26).

10. Ces éléments reviennent dans plusieurs discours, comme dans l'entretien avec Jelena*, dont le père était interprète et venait du nord de la Serbie. Entretien à son domicile le 23/04/2018, durée : 1 h18min.

* Tous les noms cités ont été anonymisés.

11. Todorova Maria, Imaginaire des Balkans, Paris, Éditions de l'EHESS, 2011, p. 282.

12. Certains travailleurs immigrés étaient logés dans des bâtiments en préfabriqué, dans le château de Montbéliard ou encore chez l'habitant, par la suite.

13. Entretien avec Jelena, fille d'un ouvrier de Peugeot, réalisé à son domicile le 23/04/2018, durée : 1 h18min.

14. Entretien avec Željko réalisé à son domicile le 25/04/2018, durée : 1h26min.

15. Entretien avec Miroslav réalisé à son domicile le 28/02/2018, durée : 15 min.

16. Voir, par exemple, l'ouvrage de Goux Jean-Paul, Mémoires de l'enclave, Arles, Actes Sud, 2003.

17. Chiffres calculés à partir du registre d'embauche du personnel de 1972. Archives de Terre Blanche, DOS 2012 ECR-05079. 
18. Notes de la sous-préfecture de Montbéliard, 2 mai 1969. Archives départementales du Doubs, 1571W79 : dossiers sur les Yougoslaves.

19. Voir l'écriteau « Yougoslaves AMAT » sur le pare-brise d'un car qui amenait les ouvriers dans leur foyer. GATTI, « Film yougoslave », op. cit.

20. Ibid.

21. D'un point de vue genré, dans un premier temps du moins, les Yougoslaves étaient essentiellement des hommes célibataires, tandis que les femmes étaient nettement minoritaires dans les recrutements de Peugeot. En 1977, par exemple, elles représentaient $12 \%$ du personnel embauché par Peugeot à Sochaux et étaient essentiellement de nationalité française.

22. MEURICE Jean-Michel, "Sochaux: 9000 célibataires", produit par l'Office national de radiodiffusion télévision française, diffusé le 9 juillet 1971.

23. Archives départementales du Territoire de Belfort, $14 \mathrm{~W}$ : demandes de permis de séjour des dossiers de demande d'étrangers.

24. Sur la place de l'automobile dans les classes populaires, voir COQUARD Benoît, "L'investissement dans la voiture compense la délocalisation des emplois », Les Jours, 30 juillet 2018.

25. Lettre datée du 3 février 1972, écrite par le Commissaire de Police, Chef du Service des Renseignements généraux et adressée au préfet de la région de Franche-Comté, préfet du Doubs. Archives départementales du Doubs, 1571W79 : dossiers sur les Yougoslaves.

26. Dans cette même lettre, nous apprenons que de faux permis de conduire auraient alors circulé.

27. Archives départementales du Territoire de Belfort, 14W : demandes de permis de séjour des dossiers de demande d'étrangers.

28. Notes de la sous-préfecture de Montbéliard, 2 mai 1969. Archives départementales du Doubs, 1571W79 : dossiers sur les Yougoslaves.

29. GATTI, « Film yougoslave », op. cit.

30. Entretien avec Ana, fille d'un ouvrier de Peugeot, réalisé à son domicile le 25/04/2018, durée : 53 minutes.

31. L'enseignement du serbo-croate était dispensé dans le Pays de Montbéliard et des villes de l'est de la France dans le cadre de l'enseignement de langue et de culture d'origine (ELCO), prévu par une directive européenne du 25 juillet 1977 «visant à la scolarisation des enfants des travailleurs migrants ».

32. VERRET, L'espace ouvrier, op. cit., p. 101-102.

33. Entretien avec Željko réalisé à son domicile le 25/04/2018, durée : $1 \mathrm{~h} 26 \mathrm{~min}$.

34. D'après l'expression employée par Armand Gatti dans son film pour désigner Sochaux.

35. Dans le dossier d'étranger de cet homme, on trouve entre autres le procès-verbal de la gendarmerie nationale précisant qu'il «n'était pas titulaire du permis de conduire les véhicules automobiles, et était dépourvu d'assurance». L'accident serait survenu "suite à une vitesse excessive, une V. L. Peugeot, circulant sens Meroux-Charmois, a dérapé dans un virage, s'est déportée sur l'accotement gauche, puis a effectué des tonneaux, pour s'immobiliser contre un arbre côté gauche sens de la marche", Archives départementales du Territoire de Belfort, 14W245 : Demandes de permis de séjour des dossiers de demande d'étrangers.

36. Voir ce qu'écrit Florence Weber sur le fait de bricoler dans une agglomération ouvrière: «Être totalement maître de son travail, voilà donc le premier sens du plaisir pris à bricoler : un pur plaisir du faire ». WEBER Florence, Le travail à-côté : étude d'ethnographie ouvrière, Paris, Éditions de l'EHESS, 1989, p. 94.

37. Entretien avec Milan et Weronika réalisé à leur domicile le 24/04/2018, durée : 2h37min.

38. Voir WEBER, Le travail à-côté, op. cit. 
39. PICHLER Robert, «Migration, Ritual and Ethnic Conflict, A Study of Wedding Ceremonies of Albanian Transmigrants from the Republic of Macedonia ", dans Klaus Roth, Jutta Lauth Bacas (dir.), Migration in, from, and to Southeastern Europe, Berlin, LIT, 2010, p. 211-229 (225).

40. Entretien avec Željko réalisé à son domicile le 25/04/2018, durée : 1h26min.

41. À Belfort, il existe une église orthodoxe - rattachée à l'archevêché des Églises orthodoxes russes du patriarcat de Moscou - particulièrement fréquentée par des personnes d'origine serbe.

42. On note par exemple l'existence d'un «club Nikola Tesla» à Kingersheim qui organise régulièrement des bals.

43. Ces enseignements s'inscrivent toujours dans le cadre de l'ELCO évoqué plus haut. Après l'éclatement de la Yougoslavie, les enseignements de serbo-croate ont été remplacés par des cours distincts de serbe et de croate.

44. À Belfort et Mulhouse, cette présence yougoslave s'explique par les activités industrielles, dont l'existence d'une autre usine Peugeot à Mulhouse, mais aussi par l'arrivée de réfugiés durant le conflit yougoslave.

45. Une association franco-serbe a été créée dans le Pays de Montbéliard en 2007 sur le modèle des associations yougoslaves qui existaient auparavant. Depuis quelques années, cette association semble en sommeil.

46. Dans le Pays de Montbéliard, on ne trouve plus que des enseignements de serbe, dispensés dans une école en dehors des horaires scolaires.

47. BEAUD Stéphane, PIALOUX Michel, «Les transformations de l'usine», Retour sur la condition ouvrière: enquête aux usines Peugeot de Sochaux-Montbéliard, Paris, La Découverte, 2012 [1999], p. 44-180.

48. BEAUD Stéphane, PIALOUX Michel, "Jeunes ouvrier(e)s à l'usine. Notes de recherche sur la concurrence garçons/filles et sur la remise en cause de la masculinité ouvrière », Travail, genre et sociétés, vol. 2, nº 8, 2002, p. 73-103.

49. Entretien avec Ana réalisé à son domicile le 25/04/2018, durée : $58 \mathrm{~min}$.

50. Entretien des époux Jovo et Zorana réalisé à leur domicile le 03/03/2018, durée : 1h27min.

51. Entretien avec Milan et Weronika réalisé à leur domicile le 24/04/2018, durée : 2h37min.

52. « Le site de PSA Sochaux en pleine mutation », L'Est républicain, 23 avril 2019.

53. VERRET, L'espace ouvrier, op. cit., p. 79.

\section{RÉSUMÉS}

Les travailleurs yougoslaves recrutés par Peugeot sont majoritairement venus en France dans les années 1960-1970 avec des expériences acquises en Yougoslavie. Cet article permet d'interroger les effets des aménagements d'une ville industrielle, marquée par le modèle paternaliste de l'entreprise Peugeot, sur les existences des ouvriers et ouvrières yougoslaves et la manière dont ces derniers et dernières se sont appropriés la ville. Ces trajectoires en France sont globalement caractérisées par d'importantes mobilités, alors que les ouvriers - hommes dans un premier temps - étaient recrutés en tant que travailleurs temporaires. L'installation de plus longue durée, pour certains, a permis le développement de pratiques spatiales centrées sur SochauxMontbéliard, malgré la perte de dynamisme de l'agglomération à partir de la fin des années 1970. Des mobilités, notamment liées aux loisirs, sont tournées vers des villes situées plus à l'est de la France et vers l'ex-Yougoslavie, durant les périodes de congés. 
The majority of Yugoslav workers recruited by Peugeot came to France in the 1960s and 1970s with experience acquired in Yugoslavia. This article examines the effects of the development of an industrial city, marked by the paternalistic model of the Peugeot company, on the lives of Yugoslav workers and the way in which they appropriated the city. These trajectories in France are characterized overall by important mobilities, whereas the workers - men at first - were recruited as temporary workers. The longer-term settlement of some of them allowed the development of spatial practices centered on Sochaux-Montbéliard, despite the loss of dynamism of the city from the end of the 1970s. Mobility, particularly for leisure purposes during vacation periods, is directed towards cities located further east in France and towards the former Yugoslavia.

INDEX

Mots-clés : Yougoslavie, France, ville, industrie, migrations, ouvriers et ouvrières

Keywords : Yugoslavia, France, city, industry, migration, workers

\section{AUTEUR}

\section{JULIETTE RONSIN}

Doctorante à l'ENS, Institut d'histoire moderne et contemporaine (IHMC)

juliette.ronsin[at]ens.fr 